\title{
Comparison of admission serum albumin and hemoglobin as predictors of outcome in children with moderate to severe traumatic brain injury \\ A retrospective study
}

\author{
Hong-chun Luo, MDª Yue-qiang Fu, MD ${ }^{b, c, d, e, f, *}$, Cheng-yan You, MD ${ }^{b, c, d, e, f}$, Cheng-jun Liu, MD ${ }^{b, c, d, e, f}$, \\ Feng $\mathrm{Xu}, \mathrm{MD}^{\mathrm{b}, \mathrm{c}, \mathrm{d}, \mathrm{e}, \mathrm{f}}$
}

\begin{abstract}
Hypoalbuminemia and anemia are frequent among in patients with traumatic brain injury (TBI). We assess whether serum albumin and hemoglobin at admission can predict outcome in children with moderate to severe TBI.

This retrospective study was conducted in a tertiary pediatric hospital between May 2012 and Jun 2018 included children with an admission Glasgow Coma Scale of $\leq 13$.

A total of 213 patients were included of whom 45 died in hospital. Multivariate logistic regression showed that hypoalbuminemia (serum albumin $<30 \mathrm{~g} / \mathrm{L}$ ) was independently associated with mortality (adjusted odds ratio [OR] =3.059; 95\% confidence interval [Cl]: 1.118-8.371; $P=.030$ ) in children with moderate to severe TBI, while anemia (hemoglobin $<90 \mathrm{~g} / \mathrm{L}$ ) was not independently associated with mortality (adjusted $\mathrm{OR}=1.742 ; 95 \% \mathrm{Cl}: 0.617-4.916 ; P=.295$ ). Serum albumin was significantly superior to hemoglobin (area under the curve [AUC] 0.738 vs AUC $0.689, P<.05$ ) under receiver operating characteristic curve analysis. Hypoalbuminemia was also associated with reduced 14-day ventilation-free days, 14-day intensive care unit (ICU)-free days, and 28day hospital-free days.

Serum albumin at admission was superior to hemoglobin in predicting the mortality in children with moderate to severe TBI and also associated with reduced ventilator-free, ICU-free, and hospital-free days.

Abbreviations: $\mathrm{AUC}=$ area under the curve, $\mathrm{Cl}=$ confidence interval, GCS = Glasgow Coma Scale, GOS = Glasgow Outcome Scale, ICU = intensive care unit, IQR = interquartile range, LOS = length of stay, OR = odds ratio, PTS = pediatric trauma score, $\mathrm{ROC}$ $=$ receiver operating characteristic, $\mathrm{TBI}=$ traumatic brain injury, $\mathrm{WBC}=$ white blood cell, WHO $=$ World Health Organization .
\end{abstract}

Keywords: albumin, hemoglobin, mortality, pediatric, traumatic brain injury

\section{Introduction}

Traumatic brain injury (TBI) is a serious cause of mortality and morbidity in children around the world. ${ }^{[1]}$ Children with moderate to severe TBI are usually in serious condition and

\section{Editor: Giovanni Tarantino.}

The authors have no conflicts of interest to disclose.

${ }^{a}$ Department of Infectious Diseases, The First Affiliated Hospital, ${ }^{b}$ Department of Critical Care Medicine, Children's Hospital, Chongqing Medical University, ${ }^{c}$ Ministry of Education Key Laboratory of Child Development and Disorders, ${ }^{a}$ National Clinical Research Center for Child Health and Disorders (Chongqing),

${ }^{e}$ China International Science and Technology Cooperation Base of Child Development and Critical Disorders, ${ }^{f}$ Chongqing Key Laboratory of Pediatrics, Chongqing, China.

*Correspondence: Yue-qiang Fu, Department of Critical Care Medicine, Children's Hospital, Chongqing Medical University, 136\# Zhongshan Er Road, Yuzhong District, Chongqing 400014, China (e-mail: fuyueqiang@sina.com).

Copyright (C) 2019 the Author(s). Published by Wolters Kluwer Health, Inc. This is an open access article distributed under the terms of the Creative Commons Attribution-Non Commercial License 4.0 (CCBY-NC), where it is permissible to download, share, remix, transform, and buildup the work provided it is properly cited. The work cannot be used commercially without permission from the journal.

How to cite this article: Luo Hc, Fu Yq, You Cy, Liu Cj, Xu F. Comparison of admission serum albumin and hemoglobin as predictors of outcome in children with moderate to severe traumatic brain injury. Medicine 2019;98:44(e17806).

Received: 8 July 2019 / Received in final form: 16 September 2019 / Accepted: 6 October 2019

http://dx.doi.org/10.1097/MD.0000000000017806 hospitalization is always needed. Besides clinical sign and imaging examination, blood measurements at admission are also important to assess the severity of TBI and predict the prognosis. Our previous study indicated that early hyperglycemia could predict for in-hospital mortality in children with moderate to severe TBI. ${ }^{[2]}$ In addition to hyperglycemia, hypoalbuminemia and anemia also occur early in patients with serious TBI.

The present studies regarding anemia and mortality in patients with TBI are conflicting. Several adult studies have shown an association between anemia and mortality in patients with TBI. ${ }^{[3-6]}$ However, other studies demonstrated no significant correlation between anemia and mortality in TBI. ${ }^{[7,8]}$ In the field of child research, Yee et $\mathrm{al}^{[7]}$ reported that anemia was not associated with hospital mortality in severe pediatric TBI patients (Glasgow Coma Scale $[\mathrm{GCS}] \leq 8$ ). Yang et al ${ }^{[8]}$ showed that initial anemia was not a mortality risk factor for patients with isolated severe blunt TBI. We should notice that the wide variations in research methodology, anemia definition and variable using of the individual studies may contribute to conflicting conclusions.

Few studies have explored the relationship between hypoalbuminemia and outcome in TBI, but previous adult studies suggested that low serum albumin was closely related to poor outcome of patients with TBI. ${ }^{[9,10]}$ Bernard et al ${ }^{[9]}$ reported that serum albumin level seemed to be an independent predictor of poor outcome (Glasgow Outcome Scale [GOS]: 1-3) in adult patients with TBI. Chen et $\mathrm{al}^{[10]}$ reported that serum albumin at 
admission could predict the poor outcome (GOS: 1-2) of adult patients with TBI.

Almost all hospitalized children with TBI are tested for serum albumin and hemoglobin. However, the predictive values of initial serum albumin or hemoglobin levels in the setting of children with TBI were rarely explored. The purpose of this retrospective casecontrol study is to investigate the predictive values of admission serum albumin and hemoglobin for in-hospital mortality of children with moderate to severe TBI and make a comparison between them. We hypothesize that serum albumin and hemoglobin at admission can predict the mortality and serum albumin may be superior to hemoglobin, and hypoalbuminemia at admission is associated with reduced ventilator-free, intensive care unit (ICU)-free, and hospital-free days.

\section{Methods}

\subsection{Ethics and consent}

The study was approved by the Medical Ethics Committee and Institutional Review Board of Children's Hospital, Chongqing Medical University and conducted in accordance with the Declaration of Helsinki. The data were analyzed anonymously. The requirement for written informed consent was waived because of the retrospective design of this study.

\subsection{Study design and patient selection}

We performed a retrospective cohort study of children with moderate to severe TBI (GCS $\leq 13$ ) admitted to ICU at the Children's Hospital of Chongqing Medical University in Chongqing, China, from May 2012 to June 2018.

We included patients younger than 16 years of age with admission GCS $\leq 13$ and abnormal head computer tomography scan at admission. We excluded the patients with diabetes mellitus, injured more than 24 hours, albumin administration or red blood cell transfusion or surgical treatment outside, hemophilia or vitamin $\mathrm{K}$ deficiency, sepsis or septic shock, massive fluid infusion before blood collection, malnutrition (body mass index Z-score $<-2$ ), and insufficient information to analyze the appropriate variables.

\subsection{Clinical variables}

The variables examined were age, gender, body weight, admission GCS score, blood glucose, serum albumin, white blood cell, hemoglobin, platelet, serum sodium, serum potassium, any other injuries in addition to the head injury, and type of injury showed in head computer tomography. Pediatric trauma score (PTS) was utilized to show the trauma severity. ${ }^{[11,12]}$ PTS was calculated by using body weight, airway status, systolic blood pressure, and consciousness level, as well as the presence of fractures or wounds on presentation. The laboratory tests were done at admission in emergency room. The length of mechanical ventilation, stay in the ICU, and in the hospital was recorded.

We defined hypoalbuminemia as albumin $<30 \mathrm{~g} / \mathrm{L}$, anemia as hemoglobin $<90 \mathrm{~g} / \mathrm{L}$, hyperglycemia as glucose $>11.1 \mathrm{mmol} / \mathrm{L}$, and hypokalemia as serum potassium $<3.5 \mathrm{mmol} / \mathrm{L}$.

\subsection{General clinical management}

The patients were maintained with the head of bed at least $30^{\circ}$ and their neck in a neutral position. Midazolam and phenobar- bital sodium were used for sedation and sufentanil for analgesia. If necessary, hyperosmolar was used to reduce intracranial pressure. Temperature was controlled to avoid hyperthermia. Neurosurgical interventions included intracranial pressure monitor, external ventricular drains, evacuation of hematoma, dissection of necrotic brain tissues and decompressive craniectomy, etc.

\subsection{Outcome}

The primary outcome was in-hospital death. Secondary outcomes were duration of mechanical ventilation, length of stay (LOS) in the ICU, and hospital. To account for death as a competing outcome, we considered the need for mechanical ventilation as ventilator-free days with a maximum of 14 days. For the LOS, we used ICU-free days and hospital-free days with a maximum of 14 and 28 days, respectively.

\subsection{Statistical analysis}

Statistical analysis was performed using the SPSS 21.0 (SPSS Inc, Chicago, IL) and (Medcalc v15.11.4, Ostend, Belgium). BMI Z-scores of children aged 0 to 5 years were calculated by World Health Organization (WHO) Anthro software, and those aged over 5 years were calculated by WHO AnthroPlus software (https://www.who.int/childgrowth/software/en/). Shapiro-Wilk analysis was used to test whether the data were normally distributed. Non-normally distributed data and categorical data was described by using median (interquartile ranges, IQR) and proportion (percentage), respectively. Comparison of 2 medians was performed by Mann-Whitney $U$ test, while the Chi-squared test was applied for categorical variables. The correlations between predictors, admission GCS, and PTS were assessed by Spearman correlation, while the correlations between glucose, hemoglobin, and serum albumin were assessed by Pearson correlation. Receiver operating characteristic (ROC) curve was used to compare the area under the curve (AUC) for serum albumin and hemoglobin as predictors of mortality. The relationship between mortality and serum albumin was also investigated with univariate and multivariate logistic regression, and anemia, hyperglycemia, hypokalemia, GCS $<8$, PTS $<8$, polytrauma, age $<36$ months, and male gender were also included in the model. $P<.05$ was considered statistically significant.

\section{Results}

A total of 288 patients were admitted during the study period, 213 of whom met the inclusion criteria for this study. The remaining 75 children were excluded due to hemophilia, receive albumin transfusion, blood transfusion, surgical treatment in other hospitals after injury, more than 24 hours after injury admitted to our hospital, or absence of blood measurements at admission.

The median age, body weight, GCS, PTS, and BMI Z score at admission of the patients were 52.0 months (IQR: 20.5-86.5), $17.0 \mathrm{~kg}$ (IQR: 12.0-25.0), 7 (IQR: 5-10), 6 (IQR: 3.5-8), and 0.41 (IQR: 0.6-1.1), respectively. The median admission serum albumin was $35.4 \mathrm{~g} / \mathrm{L}$ (IQR: 27.1-41.8) and the median hemoglobin was $98.0 \mathrm{~g} / \mathrm{L}$ (IQR: 82.5-110.5). According to the head computer tomography, $76(35.7 \%)$ patients had diffuse axonal injury, $71(33.3 \%)$ had subdural hematoma, $69(32.4 \%)$ had epidural hematoma, $106(49.8 \%)$ had subarachnoid 


\section{Table 1}

Clinical and biochemical characteristics of survivor and nonsurvivor patients.

\begin{tabular}{|c|c|c|c|}
\hline & Survivor $(n=168)$ & Nonsurvivor $(\mathrm{n}=45)$ & $P$ \\
\hline Age <36, months, $\mathrm{n}(\%)$ & 67 (39.88\%) & $17(37.78 \%)$ & .798 \\
\hline Weight $<15 \mathrm{~kg}, \mathrm{n}(\%)$ & $59(35.12 \%)$ & $21(46.67 \%)$ & .155 \\
\hline Male, n (\%) & $102(60.71 \%)$ & $25(55.56 \%)$ & .531 \\
\hline GCS $<8, \mathrm{n}(\%)$ & $72(42.86 \%)$ & $40(88.89 \%)$ & .000 \\
\hline PTS <8, n (\%) & $106(63.1 \%)$ & $42(93.3 \%)$ & .000 \\
\hline Hyperglycemia, n (\%) & $30(17.86 \%)$ & $31(68.89 \%)$ & .000 \\
\hline Serum sodium < $135 \mathrm{mmo} / \mathrm{L}, \mathrm{n}(\%)$ & $36(21.43 \%)$ & $13(28.89 \%)$ & .291 \\
\hline Hypokalemia & $60(35.71 \%)$ & $26(57.78 \%)$ & .007 \\
\hline Polytrauma, n (\%) & $74(44.05 \%)$ & $27(60.0 \%)$ & .057 \\
\hline Hypoalbuminemia, n (\%) & $38(22.62 \%)$ & $29(64.44 \%)$ & .000 \\
\hline Albumin, $(\mathrm{g} / \mathrm{L}), \mathrm{IQR}$ & $37.5(30.1-42.8)$ & $26.6(20.35-36.4)$ & .000 \\
\hline \multicolumn{4}{|l|}{ Type of injury } \\
\hline Contusion/intraparenchymal hemorrhage & $97(57.7 \%)$ & $29(64.4 \%)$ & .416 \\
\hline Brain edema & $62(36.9 \%)$ & $24(53.3 \%)$ & .046 \\
\hline Skull fracture & $114(67.9 \%)$ & $27(60.0 \%)$ & .322 \\
\hline
\end{tabular}

$\mathrm{Cl}=$ confidence interval, $\mathrm{GCS}=$ Glasgow Coma Scale, $\mathrm{IQR}=$ interquartile range, $\mathrm{PTS}=$ pediatric trauma score, $\mathrm{WBC}=$ white blood cell.

hemorrhage, $126(59.2 \%)$ had contusion/intraparenchymal hemorrhage, and $86(40.4 \%)$ had brain edema. There were $141(66.2 \%)$ patients who sustained skull/base of skull fractures.

The incidence of hypoalbuminemia and anemia at admission was $31.5 \%$ and $34.3 \%$, respectively. The in-hospital mortality rate was $21.1 \%(45 / 213)$. Comparisons of demographic and clinical severity data between the survivors and nonsurvivors were showed in Table 1. Proportions of hyperglycemia, anemia, hypokalemia, GCS $<8$, PTS $<8$, brain edema, and hypoalbuminemia were significantly higher in nonsurvivors than those in survivors.

Univariate and multivariate logistic regression analyses were performed to determine whether admission serum albumin and hemoglobin were independently associated with mortality (Table 2). Hypoalbuminemia (adjusted odds ratio $[\mathrm{OR}]=$ 3.059; 95\% confidence interval [CI]: 1.118-8.371; $P=.030)$, admission hyperglycemia (adjusted OR $=4.519$; 95\% CI: $1.865-$ $10.952 ; P=.001$ ), and GCS $<8$ (adjusted $\mathrm{OR}=6.510 ; 95 \% \mathrm{CI}$ :
1.901-22.290; $P=.003$ ) were independent risk factors for the mortality. However, we found that admission anemia (adjusted $\mathrm{OR}=1.742 ; 95 \% \mathrm{CI}: 0.617-4.916 ; P=.295)$, hypokalemia (adjusted OR $=1.499 ; 95 \% \mathrm{CI}: 0.611-3.675 ; P=.376$ ), male gender (adjusted OR $=0.416$; 95\% CI: 0.169-1.024; $P=.056$ ), age $<36$ months (adjusted OR $=0.783 ; 95 \%$ CI: $0.308-1.994$; $P=.608$ ), polytrauma (adjusted $\mathrm{OR}=0.734$; 95\% CI: $0.282-$ $1.908 ; P=.526$ ), PTS $<8$ (adjusted OR $=2.770 ; 95 \%$ CI: $0.680-$ 11.274; $P=.155$ ), and brain edema (adjusted $\mathrm{OR}=0.621 ; 95 \%$ CI: $0.243-1.586 ; P=.319$ ) were not independent risk factors for mortality in our model (Table 2).

We found a strong correlation between admission serum albumin and hemoglobin $(\mathrm{r}=0.679, P<.001)$, GCS $(\mathrm{r}=0.412$, $P<.001)$, PTS $(\mathrm{r}=0.573, P<.001)$, and glucose $(\mathrm{r}=-0.408$, $P<.001)$ through correlation analysis. However, serum albumin did not show statistically significant correlation with potassium $(\mathrm{r}=0.044, P=.522)$. In addition, admission hemoglobin also positively correlated with GCS $(\mathrm{r}=0.314, P<.001)$, PTS $(\mathrm{r}=$

Table 2

Univariate and multivariate logistic regression to identify risk factors at admission related to mortality.

\begin{tabular}{lccc}
\hline Variable & $\begin{array}{c}\text { Univariate logistic regression } \\
\text { Crude } \mathbf{~ O R ~ ( 9 5 \% ~ C I ) ~}\end{array}$ & $\boldsymbol{P}$ & $\begin{array}{c}\text { Multivariate logistic regression } \\
\text { Adjusted OR (95\% Cl) }\end{array}$ \\
\hline Anemia & $3.523(1.783-6.959)$ & .000 & $1.742(0.617-4.916)$ \\
Hyperglycemia & $10.186(4.838-21.444)$ & .000 & $4.519(1.865-10.952)$ \\
Hypokalemia & $2.463(1.260-4.816)$ & .008 & .295 \\
GCS <8 & $10.667(4.009-28.382)$ & .000 & $.009(0.611-3.675)$ \\
Hypoalbuminemia & $6.842(3.339-14.021)$ & .000 & $.510(1.901-22.290)$ \\
Male gender & $0.809(0.416-1.572)$ & .531 & $3.059(1.118-8.371)$ \\
Age <36 months & $0.915(0.465-1.801)$ & .798 & $0.416(0.169-1.024)$ \\
Polytrauma & $1.905(0.975-3.722)$ & .059 & $0.783(0.308-1.994)$ \\
PTS <8 & $8.189(2.436-27.529)$ & .001 & $0.734(0.282-1.908)$ \\
Brain edema & $1.954(1.006-3.796)$ & .048 & $2.770(0.680-11.274)$ \\
\hline
\end{tabular}

$\mathrm{Cl}=$ confidence interval, $\mathrm{GCS}=$ Glasgow Coma Scale, $\mathrm{OR}=$ odds ratio, $\mathrm{PTS}=$ pediatric trauma score. 


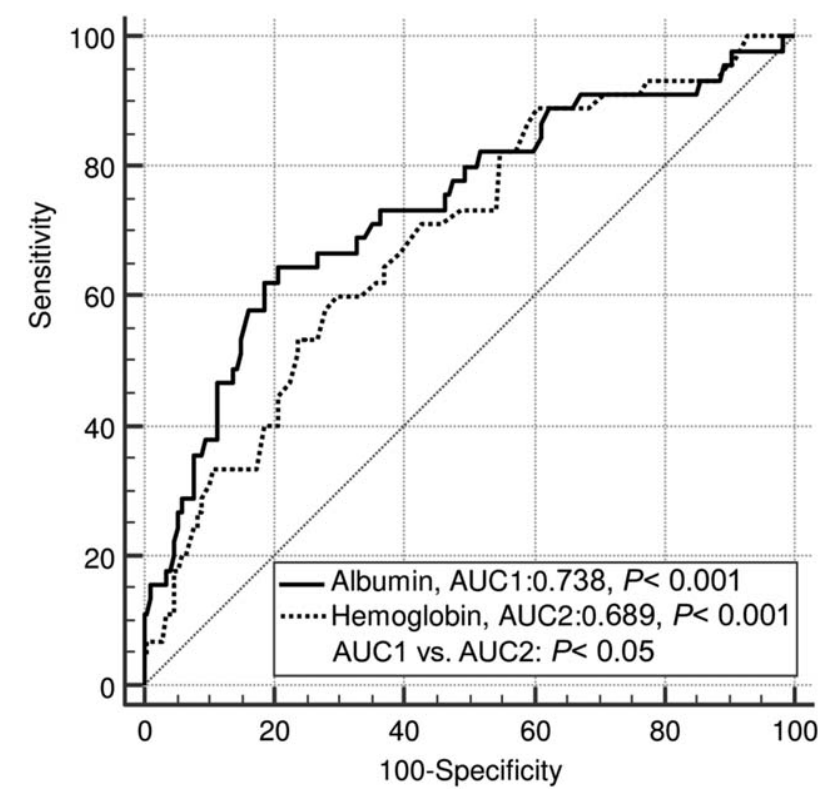

Figure 1. ROC curve analyses of mortality. The AUC of admission albumin was significantly different from the AUC of admission hemoglobin. $A \cup C=$ area under the curve, $\mathrm{ROC}=$ receiver operating characteristic.

$0.445, P<.001)$, and negatively correlated with glucose $(\mathrm{r}=-$ $0.240, P<.001)$.

AUC analysis was used to predict mortality. Admission serum albumin and hemoglobin both could predict mortality in children with moderate to severe TBI. AUC for serum albumin was of 0.738 (95\% CI: $0.673-0.796, P<.001)$ and for hemoglobin was of 0.689 (95\% CI: 0.622 to $0.750, P<.001$ ) (Fig. 1$)$. There was a significant difference between AUCs for serum albumin and hemoglobin levels $(0.738$ vs $0.689, P<.05)$.

The outcome of patients with serum albumin $<30 \mathrm{~g} / \mathrm{L}$ and $\geq 30$ $\mathrm{g} / \mathrm{L}$ were presented in Table 3. Significant difference was found between the 2 groups with respect to the mortality rate $(44.1 \%$ vs $10.3 \%, P<.001)$. Compared with patients with albumin $\geq 30 \mathrm{~g} /$ L, mechanical ventilation and ICU stay days was significantly longer, while the hospital LOS was less in patients with lactate $<30 \mathrm{~g} / \mathrm{L}$. To account for death as a competing outcome, we found that the 14 ventilation-free, 14 ICU-free, and 28 hospital-free days were all less in patients with serum albumin $<30 \mathrm{~g} / \mathrm{L}$ vs patients with serum albumin $\geq 30 \mathrm{~g} / \mathrm{L}$.

A stratified analysis of admission albumin and outcome in patients with GCS $<8$ was performed (Table 4$)$. There were still

\section{Table 3}

Clinical outcomes based on admission albumin level for patients with Glasgow Coma Scale $\leq 13$.

\begin{tabular}{lccr}
\hline & $\begin{array}{c}\text { Albumin }<\mathbf{3 0} \mathbf{g} / \mathbf{L} \\
(\mathbf{n}=\mathbf{6 8})\end{array}$ & $\begin{array}{c}\text { Albumin } \geq \mathbf{3 0 ~} \mathbf{g} / \mathbf{L} \\
(\mathbf{n}=\mathbf{1 4 5})\end{array}$ & \multicolumn{1}{c}{$\boldsymbol{P}$} \\
\hline Death, n (\%) & $30(44.1 \%)$ & $15(10.3 \%)$ & .000 \\
LOS of hospital (day) & $12.0(3.25-28.0)$ & $20.0(11.0-37.0)$ & .005 \\
28-day hospital-free days & $0(0-4.75)$ & $6.0(0-15.0)$ & $<.001$ \\
LOS of ICU (day) & $4.0(1.0-10.5)$ & $1.0(1.0-4.0)$ & .001 \\
14-day ICU-free days & $0(0-10.0)$ & $12.0(9.0-13.0)$ & $<.001$ \\
Ventilation days & $2.0(1.0-6.0)$ & $1.0(1.0-2.0)$ & $<.001$ \\
14-day ventilation-free days & $3.0(0-12.0)$ & $13.0(10.5-13.0)$ & $<.001$ \\
\hline
\end{tabular}

Data shown are number (percentage) or median (interquartile range).

$\mathrm{GCS}=$ Glasgow Coma Scale, ICU = intensive care unit, IQR = interquartile range, $\mathrm{LOS}=$ length of stay .
Table 4

Clinical outcomes based on admission albumin level for patients with Glasgow Coma Scale $<8$.

\begin{tabular}{lccc}
\hline & $\begin{array}{c}\text { Albumin }<\mathbf{3 0} \mathbf{g} / \mathbf{L} \\
(\mathbf{n = 4 8 )}\end{array}$ & $\begin{array}{c}\text { Albumin } \geq \mathbf{3 0 ~} \mathbf{g} / \mathbf{L} \\
(\mathbf{n = 6 4 )}\end{array}$ & $\boldsymbol{P}$ \\
\hline Death, n (\%) & $28.0(58.3 \%)$ & $12.0(18.8 \%)$ & .000 \\
LOS of hospital (day) & $8.0(1.0-28.0)$ & $27.0(11.25-58.75)$ & .000 \\
28-day hospital-free days & $0(0-0)$ & $0(0-6.0)$ & .019 \\
LOS of ICU (day) & $4.0(1.0-10.5)$ & $3.0(1.0-6.75)$ & .493 \\
14-day ICU-free days & $0(0-2.75)$ & $9.0(0-12.0)$ & .000 \\
Ventilation days & $4.0(1.0-7.0)$ & $1.0(1.0-4.75)$ & .020 \\
14-day ventilation-free days & $0(0-7.75)$ & $12.0(5.0-13.0)$ & .000 \\
\hline
\end{tabular}

Data shown are number (percentage) or median (interquartile range).

$\mathrm{GCS}=$ Glasgow Coma Scale, ICU = intensive care unit, IQR = interquartile range, $\mathrm{LOS}=$ length of stay

significant differences in mortality, 14 ventilator-free days, 14 ICU-free days, and 28 hospital-free days between patients with albumin $<30 \mathrm{~g} / \mathrm{L}$ vs those with albumin $\geq 30 \mathrm{~g} / \mathrm{L}$.

The relationship between admission albumin and outcome in subgroup of patients with isolated TBI $(n=112)$ was also tested. 14 ventilator-free days, 14 ICU-free days, and 28 hospital-free days of isolated TBI patients with albumin $<30 \mathrm{~g} / \mathrm{L}$ were significantly less than those of isolated TBI patients with albumin $\geq 30 \mathrm{~g} / \mathrm{L}$ (Table 5). However, on ROC analysis, admission serum albumin (AUC 0.634, 95\% CI 0.538-0.723,P=.075) and hemoglobin (AUC 0.608, 95\% CI 0.511-0.698, $P=.082$ ) both could not predict mortality in children with isolated moderate to severe TBI.

It was worth noting that compared with TBI patients with polytrauma $(\mathrm{n}=101)$, patients with isolated TBI had significantly higher GCS (9.0 vs $6.0, P<.001)$ and PTS $(7.0$ vs $5.0, P<.001)$. The incidence of hypoalbuminemia in patients with isolated TBI is significantly lower than that in TBI patients with polytrauma $(20.5 \%$ vs $44.6 \%, P<.001)$. Moreover, the mortality rate of patients with isolated TBI was also lower than that of TBI patients with polytrauma, but the difference failed to reach statistical significance $(16.1 \%$ vs $26.7 \%, P=.057)$.

\section{Discussion}

The main findings of this study are the following: serum albumin and hemoglobin at admission both can predict in hospital mortality on ROC curve analysis; however, serum albumin is a better predictor than hemoglobin in determining the risk of mortality for children with moderate to severe TBI; there was a

\section{Table 5}

Clinical outcomes based on admission albumin level for patients with isolated traumatic brain injury.

\begin{tabular}{lccc}
\hline & $\begin{array}{c}\text { Albumin }<\mathbf{3 0} \mathbf{g} / \mathbf{L} \\
(\mathbf{n}=\mathbf{2 3})\end{array}$ & $\begin{array}{c}\text { Albumin } \geq \mathbf{3 0} \mathbf{g} / \mathbf{L} \\
(\mathbf{n}=\mathbf{8 9})\end{array}$ & $\boldsymbol{P}$ \\
\hline Death, n (\%) & $8(34.8 \%)$ & $10(11.2 \%)$ & .006 \\
LOS of hospital (day) & $12.0(6.0-30.0)$ & $16.0(10.0-26.5)$ & .362 \\
28-day hospital-free days & $0(0-11.0)$ & $8.0(0-16.0)$ & .020 \\
LOS of ICU (day) & $2.0(1.0-7.0)$ & $1.0(1.0-3.0)$ & .067 \\
14-day ICU-free days & $7.0(0-13.0)$ & $13.0(9.0-13.0)$ & .006 \\
Ventilation days & $1.0(1.0-6.0)$ & $1.0(1.0-1.5)$ & .020 \\
14-day ventilation-free days & $9.0(0-13.0)$ & $13.0(11.5-13.0)$ & .002 \\
\hline
\end{tabular}

Data shown are number (percentage) or median (interquartile range).

$\mathrm{GCS}=$ Glasgow Coma Scale, ICU = intensive care unit, IQR = interquartile range, $\mathrm{LOS}=$ length of stay . 
strong positive correlation between serum albumin and hemoglobin, and these 2 variables also positively correlated with admission GCS and PTS; on multivariate regression analysis, hypoalbuminemia was independently associated with mortality, whereas anemia was not an independent risk factor; hypoalbuminemia was associated with reduced 14-day ventilation-free days, 14-day ICU-free days, and 28-day hospital-free days. Therefore, our study indicates that serum albumin at admission should be preferred to hemoglobin as a biologic variable in predicting in hospital mortality.

Our study shows the incidence of hypoalbuminemia $(31.5 \%)$ and anemia $(34.3 \%)$ at admission is relatively high. However, only very few studies were involved in serum albumin or hemoglobin in pediatric patients with TBI. ${ }^{[4,7,13]}$ The level of serum albumin can reflect the severity of TBI. Chen et al ${ }^{[10]}$ reported that serum albumin was positively correlated with GCS in adult patients with TBI. Our data show that admission albumin was positively correlated with admission GCS, PTS, and hemoglobin, and negatively correlated with admission glucose. In addition, hemoglobin was also positively correlated with GCS and PTS, and negatively correlated with glucose. These findings indicate that low levels of admission albumin and hemoglobin were associated with severe head injury and elevated levels of glucose.

Hypoalbuminemia is common in critically ill patients. Some studies showed that serum albumin at admission was associated with mortality. ${ }^{[14-17]}$ One study found that serum albumin at admission could be used as a sensitive and specific marker of burn severity and an indicator of mortality in adult patients with burn. ${ }^{[14]}$ Hypoalbuminemia at admission was independently associated with higher mortality at 90 days in acute intracerebral hemorrhage. ${ }^{[15]}$ Sung et $\mathrm{al}^{[16]}$ reported that $42 \%$ patients were admitted with a serum albumin level of $<2.6 \mathrm{~g} / \mathrm{dL}$, the admission serum albumin level of $<2.6 \mathrm{~g} / \mathrm{dL}$ is a significant independent predictor of morbidity and mortality in trauma patients. Leite et $\mathrm{a}^{[17]}$ found that hypoalbuminemia at admission to a pediatric intensive care unit (PICU) was associated with higher 60-day mortality.

Albumin constitutes up to two-third of total plasma protein, contributing about $80 \%$ of the plasma colloid osmotic pressure and is responsible for the transport and binding of many molecules. There are several possible causes of hypoalbuminemia at admission in TBI. Firstly, the loss of serum albumin in TBI may be attributed to hemorrhage. Trauma leads to vascular damage, including blood-brain barrier, which causes loss of albumin and hemoglobin. To support this view, we find a significantly positive correlation between albumin and hemoglobin levels at admission. Secondly, strong stress following TBI may also result in massive release of inflammatory mediators which enhance vascular permeability. Enhanced vascular permeability would encourage a larger shift of albumin from the vascular to the interstitial space. ${ }^{[18-20]}$ Thirdly, stress may also increase albumin consumption. Whatever the cause of hypoalbuminemia, the decreased plasma colloid osmotic pressure compromises the intravascular volume, placing the child at risk for inadequate blood flow to vital organs. Worsening hypoalbuminemia is likely to cause secondary brain injury and contribute to the mortality and morbidity in TBI.

In the field of TBI research, studies have shown that serum albumin could be used as a biomarker to predict the prognosis of patients with TBI. Chen et $\mathrm{al}^{[10]}$ showed that serum albumin within 24 hours after admission could predict poor outcomes (GOS: 1-2) in patients with severe TBI. Schirmer-Mikalsen et $\mathrm{al}^{[21]}$ reported that hypoalbuminemia (albumin $<25 \mathrm{~g} / \mathrm{L}$ ) during the first day in ICU/high-dependency unit was possible independent predictors of an unfavorable outcome (GOS: 1-3) in patients with severe head injury. Other previous studies also showed that serum albumin seemed to be an independent predictor for poor outcome of TBI. ${ }^{[9,22]}$ Our findings indicate that in children with moderate to severe TBI, serum albumin at admission is a reliable indicator of those who are at the risk of in hospital death (adjusted OR: 3.140; 95\% CI: 1.242-7.936).

Some studies indicated that serum albumin was associated with the length of ventilation and hospital stay. ${ }^{[16,23]}$ Hypoalbuminemia at admission to a PICU was associated with longer duration of mechanical ventilation, and lower probability of ICU discharge. ${ }^{[16]}$ Chung et al ${ }^{[23]}$ reported that low albumin levels before surgery were associated with significantly longer lengths of stay in patients undergoing hip fracture surgery. In this study, we find that 14 ventilation-free, 14 ICU-free, and 28 hospital-free days of the patients with hypoalbuminemia are less than those of the patients with albumin $\geq 30 \mathrm{~g} / \mathrm{L}$ in all patients, subgroup of patient with GCS $<8$, and subgroup of patients with isolated TBI, respectively. Meanwhile, the mortality rates of patients with hypoalbuminemia are also significantly higher than those of the patients with albumin $\geq 30 \mathrm{~g} / \mathrm{L}$ in all above 3 groups, respectively. Therefore, we argue that hypoalbuminemia is associated with death, reduced ventilator-free, ICU-free, and hospital-free days in children with moderate to severe TBI.

Hemoglobin is the major carrier of oxygen in the systemic circulation. Anemia following TBI will reduce the oxygencarrying capacity of the blood, which may contribute to secondary brain injury after trauma. Oddo et $\mathrm{al}^{[24]}$ reported that hemoglobin $<90 \mathrm{~g} / \mathrm{L}$ was associated with lower brain tissue oxygen tension $\left(\mathrm{PbtO}_{2}\right)$, and anemia combined with reduced $\mathrm{PbtO}_{2}$ was associated with unfavorable outcome after TBI. Our study shows that hemoglobin at admission can predict mortality on ROC curve analysis, but AUC was smaller for hemoglobin than for albumin. Furthermore, we find that anemia is not an independent risk factor of mortality. The result is not surprising. In theory, worsening anemia after trauma is certainly harmful to the human body. However, the researches on the relationship between anemia and the prognosis of patients with TBI are full of controversy at present. Some studies showed worse outcomes with anemia, ${ }^{[3-6]}$ whereas others showed no detrimental effect. ${ }^{[7,8,25]}$ Litofsky et al ${ }^{[3]}$ found that initial hemoglobin was independently associated with poor outcome in patients with TBI. Sekhon et $\mathrm{al}^{[4]}$ reported that a mean hemoglobin level $<90 \mathrm{~g} /$ $\mathrm{L}$ during the first week of hospitalization increased risk for inhospital mortality in patients with severe TBI. Salim et $\mathrm{al}^{[6]}$ reported that hemoglobin $<90 \mathrm{~g} / \mathrm{L}$ was a significant risk factor for mortality when transfusion was not included in adult TBI patients. However, some studies demonstrated no significant correlation between anemia and poor outcome. ${ }^{[7,8,25]}$ Yee et $\mathrm{al}^{[7]}$ reported that neither anemia nor blood transfusion was associated with hospital mortality in pediatric patients with TBI. Yang et $\mathrm{al}^{[8]}$ showed that initial anemia (hemoglobin $<10 \mathrm{mg} / \mathrm{dL}$ ) was not a risk factor for mortality in patients with isolated severe blunt TBI. Okoye et $\mathrm{al}^{[25]}$ reported that anemia (hemoglobin $8 \mathrm{~g} / \mathrm{dL}$ ) was not associated with increased mortality or complications in patients with TBI. Of course, these studies, including our research, were all retrospective and had their own limitations. Prospective studies are needed to explore the relationship between anemia and mortality in TBI. 
On ROC analysis, admission serum albumin and hemoglobin both could predict mortality in all patients with moderate to severe TBI. However, in the subgroup of patients with isolated TBI, both admission serum albumin (AUC 0.634, 95\% CI 0.538$0.723, P=.075$ ) and hemoglobin (AUC 0.608, 95\% CI 0.511$0.698, P=.082$ ) show no predictive value. We argue that polytrauma can aggravate the severity of children with TBI and increase the incidence of hypoalbuminemia, although it is not an independent risk factor for mortality. Removal of patients with polytrauma results in weakening the predictive value of admission serum albumin for mortality. Frankly, this is 1 singlecenter retrospective study and the predictive value of admission serum albumin for mortality in children with moderate to severe TBI needs further multicenter researches to explore.

In additional to physical examination and imaging examination, blood test is also an important method to assess the condition of patients with TBI. Some of blood parameters, including albumin and hemoglobin, can guide resuscitation and predict outcome after injury. Although serial measurements may be optimal as part of a goal-directed resuscitation, blood parameters at admission still have the advantage of providing doctors with key information in time and helping doctors take relevant treatments quickly. The present study is the first study which focuses on the comparison of postinjury albumin and hemoglobin at admission in children with moderate to severe TBI. Our data demonstrate that serum albumin at admission may be used to assess the degree of injury severity and predict the prognosis.

We recognize several limitations in this study. Firstly, it is a retrospective designed study from only 1 medical center. Secondly, the study population is relatively small. Thirdly, the study was not designed to evaluate the impact of albumin administration and red blood cell transfusion on the outcome of children with TBI. We only studied the impact of blood biomarkers collected at the time of admission on the prognosis of patients. Red blood cell transfusions seem to be a stronger death contributor than anemia itself. ${ }^{[6,26]}$ Similar to the impact of blood transfusion, 2 studies showed albumin administration was associated with poor outcome in TBI. ${ }^{[27,28]}$ The impact of albumin administration on the prognosis of children with moderate to severe TBI need further explore. Finally, there is no uniform definition of hypoalbuminemia or anemia in trauma studies. The definitions of hypoalbuminemia and anemia in our study may be inconsistent with other trauma studies. Despite these limitations, we find that serum albumin at admission have a predictive and prognostic value, which enables clinicians to identify early, children with moderate to severe TBI who are at great risk of in hospital mortality.

\section{Conclusions}

Serum albumin at admission is superior to hemoglobin in predicting the risk of mortality. Furthermore, hypoalbuminemia is an independent risk factor for mortality on multivariate regression. Hypoalbuminemia is associated with reduced ventilator-free, ICU-free, and hospital-free days. We conclude that serum albumin at admission is a useful biomarker for children with moderate to severe TBI.

\section{Author contributions}

Conceptualization: Yue-qiang Fu, Feng Xu.
Data curation: Hong-chun Luo, Yue-qiang Fu, Cheng-yan You, Cheng-jun Liu.

Formal analysis: Hong-chun Luo, Yue-qiang Fu, Cheng-yan You, Cheng-jun Liu.

Investigation: Hong-chun Luo, Yue-qiang Fu.

Project administration: Yue-qiang Fu.

Resources: Yue-qiang Fu, Hong-chun Luo.

Software: Hong-chun Luo, Yue-qiang Fu, Cheng-yan You, Cheng-jun Liu.

Supervision: Yue-qiang Fu.

Validation: Yue-qiang Fu.

Writing - original draft: Hong-chun Luo.

Writing - review \& editing: Yue-qiang Fu, Feng $\mathrm{Xu}$.

\section{References}

[1] Anderson VA, Catroppa C, Haritou F, et al. Identifying factors contributing to child and family outcome 30 months after traumatic brain injury in children. J Neurol Neurosurg Psychiatry 2005;76: 401-8.

[2] Fu YQ, Chong SL, Lee JH, et al. The impact of early hyperglycaemia on children with traumatic brain injury. Brain Inj 2017;31:396-400.

[3] Litofsky NS, Martin S, Diaz J, et al. The negative impact of anemia in outcome from traumatic brain injury. World Neurosurg 2016;90:82-90.

[4] Sekhon MS, McLean N, Henderson WR, et al. Association of hemoglobin concentration and mortality in critically ill patients with severe traumatic brain injury. Crit Care 2012;16:R128.

[5] Duane TM, Mayglothling J, Grandhi R, et al. The effect of anemia and blood transfusions on mortality in closed head injury patients. J Surg Res 2008;147:163-7.

[6] Salim A, Hadjizacharia P, DuBose J, et al. Role of anemia in traumatic brain injury. J Am Coll Surg 2008;207:398-406.

[7] Yee KF, Walker AM, Gilfoyle E. The effect of hemoglobin levels on mortality in pediatric patients with severe traumatic brain injury. Can Respir J 2016;2016:6803860.

[8] Yang CJ, Hsiao KY, Su IC, Chen IC. The association between anemia and the mortality of severe traumatic brain injury in emergency department. J Trauma 2011;71:E132-5.

[9] Bernard F, Al-Tamimi YZ, Chatfield D, et al. Serum albumin level as a predictor of outcome in traumatic brain injury: potential for treatment. J Trauma 2008;64:872-5.

[10] Chen D, Bao L, Lu SQ, Xu F. Serum albumin and prealbumin predict the poor outcome of traumatic brain injury. PLoS One 2014;9: e93167.

[11] Tepas JJ3rd, Mollitt DL, Talbert JL, et al. The pediatric trauma score as a predictor of injury severity in the injured child. J Pediatr Surg 1987;22:14-8.

[12] Tepas JJ3rd, Ramenofsky ML, Mollitt DL, et al. The Pediatric Trauma Score as a predictor of injury severity: an objective assessment. J Trauma 1988;28:425-9.

[13] Kamal H, Mardini A, Aly BM. Traumatic brain injury in pediatric age group; predictors of outcome in Pediatric Intensive Care Unit. Libyan J Med 2007;2:90-4.

[14] Aguayo-Becerra OA, Torres-Garibay C, Macías-Amezcua MD, et al. Serum albumin level as a risk factor for mortality in burn patients. Clinics (Sao Paulo) 2013;68:940-5.

[15] Morotti A, Marini S, Lena UK, et al. Significance of admission hypoalbuminemia in acute intracerebral hemorrhage. J Neurol 2017; 264:905-11.

[16] Sung J, Bochicchio GV, Joshi M, et al. Admission serum albumin is predictive of outcome in critically ill trauma patients. Am Surg 2004;70:1099-102.

[17] Leite HP, Rodrigues da Silva AV, de Oliveira Iglesias SB, et al. Serum albumin is an independent predictor of clinical outcomes in critically ill children. Pediatr Crit Care Med 2016;17:e50-7.

[18] Corrigan F, Mander KA, Leonard AV, Vink R. Neurogenic inflammation after traumatic brain injury and its potentiation of classical inflammation. J Neuroinflammation 2016;13:264.

[19] Sorby-Adams AJ, Marcoionni AM, Dempsey ER, et al. The role of neurogenic inflammation in blood-brain barrier disruption and development of cerebral oedema following acute central nervous system (CNS) injury. Int J Mol Sci 2017;18: 
[20] Rodoman GV, Shalaeva TI, Dobretsov GE, et al. Serum albumin in systemic inflammatory reaction syndrome. Anesteziol Reanimatol 2006; $2: 62-4$.

[21] Schirmer-Mikalsen K, Vik A, Gisvold SE, et al. Severe head injury: control of physiological variables, organ failure and complications in the intensive care unit. Acta Anaesthesiol Scand 2007;51:1194-201.

[22] Nelson DW, Rudehill A, MacCallum RM, et al. Multivariate outcome prediction in traumatic brain injury with focus on laboratory values. J Neurotrauma 2012;29:2613-24.

[23] Chung AS, Hustedt JW, Walker R, et al. Increasing severity of malnutrition is associated with poorer 30-day outcomes in patients undergoing hip fracture surgery. J Orthop Trauma 2018;32:155-60.

[24] Oddo M, Levine JM, Kumar M, et al. Anemia and brain oxygen after severe traumatic brain injury. Intensive Care Med 2012;38:1497-504.
[25] Okoye O, Inaba K, Kennedy M, et al. The impact of anemia in moderate to severe traumatic brain injury. Eur J Trauma Emerg Surg 2013;39: 627-33.

[26] Al-Dorzi HM, Al-Humaid W, Tamim HM, et al. Anemia and blood transfusion in patients with isolated traumatic brain injury. Crit Care Res Pract 2015;2015:672639.

[27] Cooper DJ, Myburgh J, Heritier S, et al. Albumin resuscitation for traumatic brain injury: is intracranial hypertension the cause of increased mortality? J Neurotrauma 2013;30:512-8.

[28] Myburgh J, Cooper DJ, Finfer S. SAFE Study Investigators; Australian and New Zealand Intensive Care Society Clinical Trials Group; Australian Red Cross Blood Service; George Institute for International HealthSaline or albumin for fluid resuscitation in patients with traumatic brain injury. N Engl J Med 2007;357:874-84. 